\title{
Automated Detection of ECG Noise Signal and Classification System by Using Modified CEEMD
}

\author{
A.Rajani | B.Prasanna Lakshmi
}

Electronics and Communication Engineering, JNTUK, Kakinada, Andhra Pradesh, India

To Cite this Article

A.Rajani and B.Prasanna Lakshmi, "Automated Detection of ECG Noise Signal and Classification System by Using Modified CEEMD”, International Journal for Modern Trends in Science and Technology, 6(8S): 128-133, 2020.

\section{Article Info}

Received on 16-July-2020, Revised on 15-August-2020, Accepted on 25-August-2020, Published on 28-August-2020.

\section{ABSTRACT}

Electrocardiogram (ECG) depicts the electrical activity of heart. If the signal obtained is not proper then wrong analysis might be done. Due to which wrong diagnosis could be done. So there is a need to extract clean ECG signal. Electrocardiogram (ECG) signals are often corrupted with different types of noises and artifacts such as baseline wander and drift (electrode contact noise and electrode motion artifacts), powerline interference (PLI), and muscle artifacts, making it almost impossible to perform a morphological and RR interval analysis of such contaminated ECG signals. The proposed framework consists of the modified ensemble empirical mode decomposition (CEEMD), the short-term temporal feature extraction, and the decision rules-based noise detection and classification the proposed framework, ECG signals are first decomposed using the modified CEEMD algorithm for discriminating the ECG components from the noises and artifacts. Then, the short-term temporal features such as maximum absolute amplitude, number of zerocrossings, and local maximum peak amplitude of the autocorrelation function are computed from the extracted high-frequency (HF) and low-frequency (LF) signals. Finally, a decision rule-based algorithm is presented for detecting the presence of noises and classifying the processed ECG signals into six signal groups: noise-free ECG, $E C G+B W, E C G+M A, E C G+P L I, E C G+B W+P L I$, and $E C G+B W+M A$. In the proposal method a modified Complete Ensemble Empirical Mode Decomposition (CEEMD) algorithm with new stopping criteria is proposed which reduces this computational load significantly and greatly reducing the no of false alarm rate.

KEYWORDS: ECG Signals, Empirical Mode Decomposition, Eigen Value Decomposition, IMF

\section{INTRODUCTION}

Electrocardiogram (ECG) signals are often corrupted with different types of noises and artifacts, such as baseline wan- der and drift (electrode contact noise and electrode motion artifacts), powerline interference (PLI), and muscle artifacts, making it almost impossible to perform a morphological and RR interval analysis of such contaminated ECG signals. Most of the existing ECG analysis systems were designed to handle relatively noise-free ECG signals. In such scenarios, existing systems render the inaccurate and unreliable measurements which lead to produce high false alarm rates for the noisy ECG signals. Consequently, frequent false alarms are not only most annoying and disturbing both the clinicians and the patients but also lead to misdiagnosis of cardiac arrhythmias. The issue of high false arrhythmia event alarm and heart-rate alarm rates highly impacts the usability of real-time ECG monitors because of the two main reasons: 
(i) noises and artifacts in the isoelectric line of the ECG signal are falsely detected as normal beats or abnormal beats; and (ii) severely contaminated ECG beats are misclassified due to the inaccurate measurements of essential ECG

.Electrocardiogram (ECG) signals are widely acquired for many applications such as cardiovascular disease diagnosis, arrhythmias recognition, physiological feedback, sleep apnea detection, chronic patient surveillance, sudden cardiac arrest prediction, biometric, emotionaland physical activity recognition systems. All of these ECG application systems highly demand the exact determination of fiducial points or (characteristic points) of the ECG signal for accurate and reliable measurements of morphological features (including, amplitude, duration, polarity and shape) of local waves such as P-wave, QRScomplex, T-wave and U-wave, and the interval features (including, PR-segment, QT-segment, STsegment, RR interval). Most ECG analysis systems require relatively noise-free ECG signals for obtaining the ECG measurements more accurately and reliably. In practice, the ECG signals are often corrupted with different types of noises such as baseline wander (electrode contact noise and electrode motion artifacts), power line interference (PLI), electromyogram (EMG) noise, and instrumentation (IN) noise, making it almost impossible to perform a morphological analysis of such contaminated ECG beats. Therefore, automatic assessment of ECG signal is highly demanded in reducing the false alarms due to presence of unacceptable level of noises.

\section{A.Related Work}

Various strategies have been adopted to handle the problem of high false alarm rate due to the noises and artifacts:

(i) ECG denoising based approach to suppress the noises and artifacts in the ECG recordings and (ii) signal quality indices (SQI) based approach to assess the clinical acceptability of the recorded ECG signals.

1) ECG Denoising Based Strategy: To reduce the false alarm rates, various ECG denoising methods have been presented based on the moving average and median filters, frequency-selective filters, adaptive filters, Wiener filters, polynomial filters, singular value decomposition (SVD), discrete cosine transform (DCT), discrete wavelet transform(DWT), switching Kalman filters, empirical mode decomposition (EMD),nonlinear
Bayesian filter (NBF), mathematical morphological (MM) operators, principal component analysis (PCA), independent component analysis(ICA), nonlocal means (NLM) method, variational mode decomposition (VMD), and EMD-wavelet method for removal of single and combined ECG noise sources. Most existing de- noising methods are highly capable of suppressing noises and artifacts that are very different from those of ECG morphology. Evaluation results show that the baseline wander removal methods may distort the ST-segment due to the attenuation of the low-frequency components of ECG signal. In signal decomposition-based methods, ECG signal is decomposed (using empirical mode decomposition and its variants) into number of intrinsic mode functions (IMFs) and then different statistical features are extracted fromthese IMFs for detection.

2) Signal Quality Assessment Based Strategy: Besides the noise reduction strategy, signal quality assessment (SQA) strategy has been employed to tackle the false alarm problem. Most methods were presented for classifying the recorded ECG signals into acceptable or unacceptable. Most methods were developed based on the QRS complex detectors and the ECG morphological and interval features that rely on accurate and reliable determination of R-peaks in the ECG signals.

\section{B. Objective and Key Contributions}

A single signal processing technique isnot adequate to remove different kinds of noises and artifacts in the ECG signals. Moreover, the denoising results show that each of the filtering techniques introduce different kinds of waveform distortion. Thus, it is very important to identify the nature of noises in the ECGrecordings and to choose appropriate signal processing techniques suited to the noise types.

There is no systematic framework which can perform automatic detection, localization and classification of noises and artifacts present in the ECGsignal.Theseissuesareveryimportantdesign consideration for real-time ECG signal analysis and diagnosis systems under resting, ambulatory and exercise ECG recording conditions.

\section{Procedure}

This paper proposes a novel unified framework for automatic detection, localization and classification of noises and artifacts present in the ECG signal 
and finally removing of noise to improve the quality of signal. The present study has four major goals:

(i) Unlike past SQA studies, we aim to propose an unified ECG noise detection, localization and classification framework, which has great potential in reducing the number of false alarm rates and selecting noise-specific signal processing (or noise models) technique(s) for effective removal of noises from ECG signal.

(ii) We intend to reduce computational load of the commonly used CEEMD algorithm by introducing new stopping criteria such as number of zero crossings (NZC) and maximum absolute amplitude (MAA) in the iteration process.

(iii)We explore short-term temporal feature extraction for detection, localization and classification of baseline wander (BW), muscle artifacts (MA), and powerline interference (PLI).

(iv) We build decision rules based algorithm for classifying the ECG signals into six groups: noise-free ECG, ECG+BW, ECG+MA, ECG+PLI, $\mathrm{ECG}+\mathrm{BW}+\mathrm{PLI}$, and $\mathrm{ECG}+\mathrm{BW}+\mathrm{MA}$. Evaluation results demonstrate that the proposed framework achieves higher sensitivity, positive predictivity, andaccuracy in detection and classification of noises and artifacts present in the ECG signal.

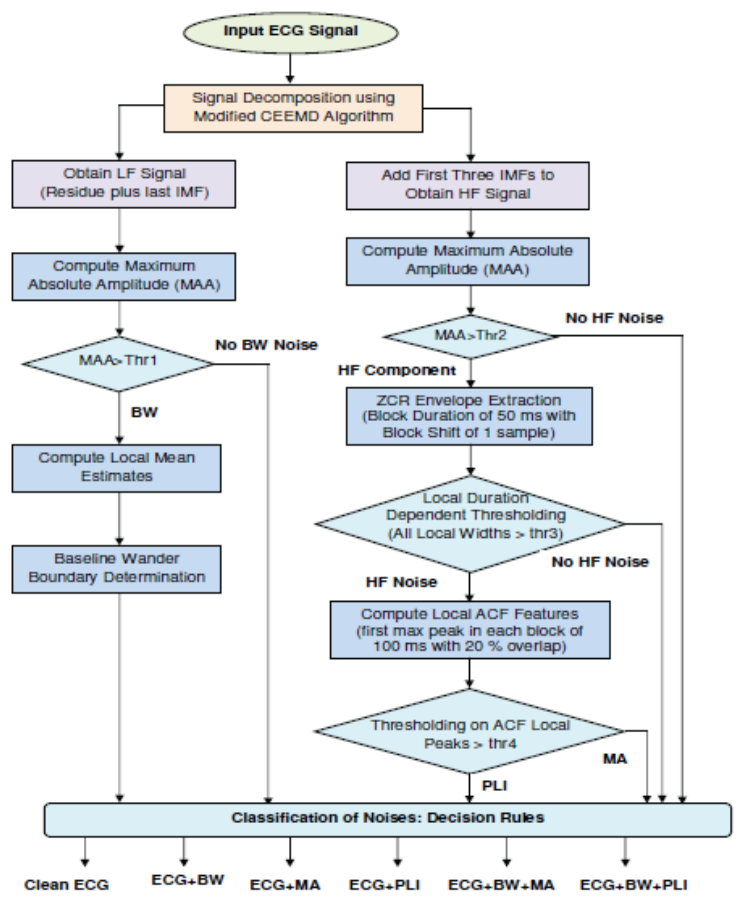

Fig 1. Flow of Proposed Methodology
Finally Baseline wander and power line interference removal from ECG signals using eigenvalue decomposition is obtained and the results are shown.

\section{Modified CEED Algorithm}

There are several techniques to decompose an ECG signal into several sub signals. Empirical mode decomposition (EMD), which is a self-adaptive time-frequency analysis technique, is widely used for decomposing complex, multicomponent signal into several fast and slow oscillations called intrinsic mode functions (IMFs). The complete ensemble EMD technique has been proposed to overcome the drawbacks of the basic EMD and ensemble EMD (EEMD), such as mode mixing problem of the basic EMD, where different oscillations exist in the same IMF, or similar oscillations exist in different IMFs; producing varying number of IMFs; and reconstructed signals contain residual noise after decomposition when signal to noise ratio (SNR) is low. Torres et al. proposed the CEEMD algorithm that adds different realizations of Gaussian noise to the residual signal after extracting subsequent intrinsic mode functions. It has been proven that the CEEMD algorithm provides an exact reconstruction of the original signal andan improved spectral partition of the modes, with a lower computational cost by requiring less than half the sifting iterations that for the EEMD algorithm. By applying the CEEMD algorithm, a signal is adaptively decomposed into a finite set of intrinsic mode functions (or oscillation modes)andaresidue, whereinthedecompositionproc edure is continued until the obtained residue is no longer feasible to be decomposed. The lower-order IMFs capture fast oscillation modes of the high-frequency noises caused by muscle artifacts, power line interferences, and recording instrument noises while higher- order IMFs typically capture slow oscillation modes of base- line wanders.

In the modified CEEMD algorithm, the decomposition process is terminated when any following stopping criterion is satisfied, namely, the magnitude of the current residue is less than the predefined threshold, or the number of zero crossings (NZC) of the current residue is less than the predefined NZC value. The result of the modified CEEMD algorithm produces a finite set of IMFs and a residue of baseline wander. The predefined threshold values for the stopping criteria are chosen based on the temporal parameters such as maximum absolute amplitude 
and number of zerocrossings of the slow oscillation of the baseline wanders. By considering the baseline wanders with frequency less than $1 \mathrm{~Hz}$ and the magnitude of severe baseline wanders, the NZC of 10 and MAA of $0.1 \mathrm{mV}$ are chosen to discriminate the baseline wanders from the ECG components and the MA and PLI noises.

For a given input ECG signal $\mathrm{x}[\mathrm{n}]$, the modified CEEMDalgorithm with new stopping criteria can be summarized as:

1) Obtain different realizations of signal plus white Gaussian noisew ${ }^{k}[n]$ of standard deviation $\epsilon$ i.e., $x^{k}[n]=x[n]+w^{k}[n]$

2) Decompose the signal plus noise realizations $x[n]$ $+\in \circ w^{k}[n]$ using EMD to get their first IMFs and then compute the first IMF as

$$
\overline{I M F_{1}}[n]=\frac{1}{K} \sum_{k=1}^{k} I M F_{1}^{k}[n]
$$

3) Compute first residue as $r_{1}[\mathrm{n}]=\mathrm{x}[\mathrm{n}]-\overline{I M F_{1}}$

4) Decompose realizations $r_{1}[n]+\epsilon_{1} w^{k}[n]$ to get their firstEMD mode and obtain the second mode:

$$
\overline{I M F_{2}}[n]=\frac{1}{K} \sum_{k=1}^{k} E_{1}\left(\mathrm{r}_{1}[n]+\epsilon_{1} w^{k}[n]\right)(2)
$$

where $E_{1}$ denotes first mode from EMD decomposition.

5) For finding the $i-t h$ mode, decompose realizations of the $i-t h$ residue, $r_{i}[n]+\epsilon_{i} w^{k}[n]$ to get their first EMD Mode and obtain the $(i+1)-t h$ node as

$$
\overline{I M F_{i+1}}[n]=\frac{1}{K} \sum_{k=1}^{k} E_{1}\left(\mathrm{r}_{i}[n]+\epsilon_{i} w^{k}[n]\right)
$$

$$
i=1,2 \ldots I
$$

where I denotes the total number of modes andand $\gamma_{i}(n)=\gamma_{(i-1)}(n) \mid-\overline{\mathrm{IMF}_{i}}(\mathrm{n})$ is the residue for $i-t h$ mode.

6) Compute the NZC and the MAA for the $\overline{I M F_{i}}(n)$.

7) Continue step 5 for next mode until the obtained residue does not satisfy the proposed stopping criteria: if $(\mathrm{NZC}>10||$ MAA $>0: 1)$ is true.

In this study, one of the goals is to improve the computationalload of the CEEMD algorithm.

\section{Signal and Noise Separation}

The decomposition results of the modified CEEMD algorithm are shown in Fig. 2. Results show that lower-order IMFs and higher-orderIMFs capture the fine scales (or high-frequency (HF) components) and coarse scales (or low frequency (LF) components) of the ECG signals, respectively. For the noise-free ECG signals, lower-order IMFs, or modes M1 and M2 in Fig. 2, capture fast varyingcomponentsofQRScomplexesandhigherorder IMFs, or modes M8 and M9 in Fig. 2, capture very LF componentsof the local waves For the noisy ECG signals, lower-order IMFs capture the HF components of the MA, PLI noises and the HF componentsof QRS complexes. For both cases, the final residue includes the components of the baselinewanders.

\section{.Figures}

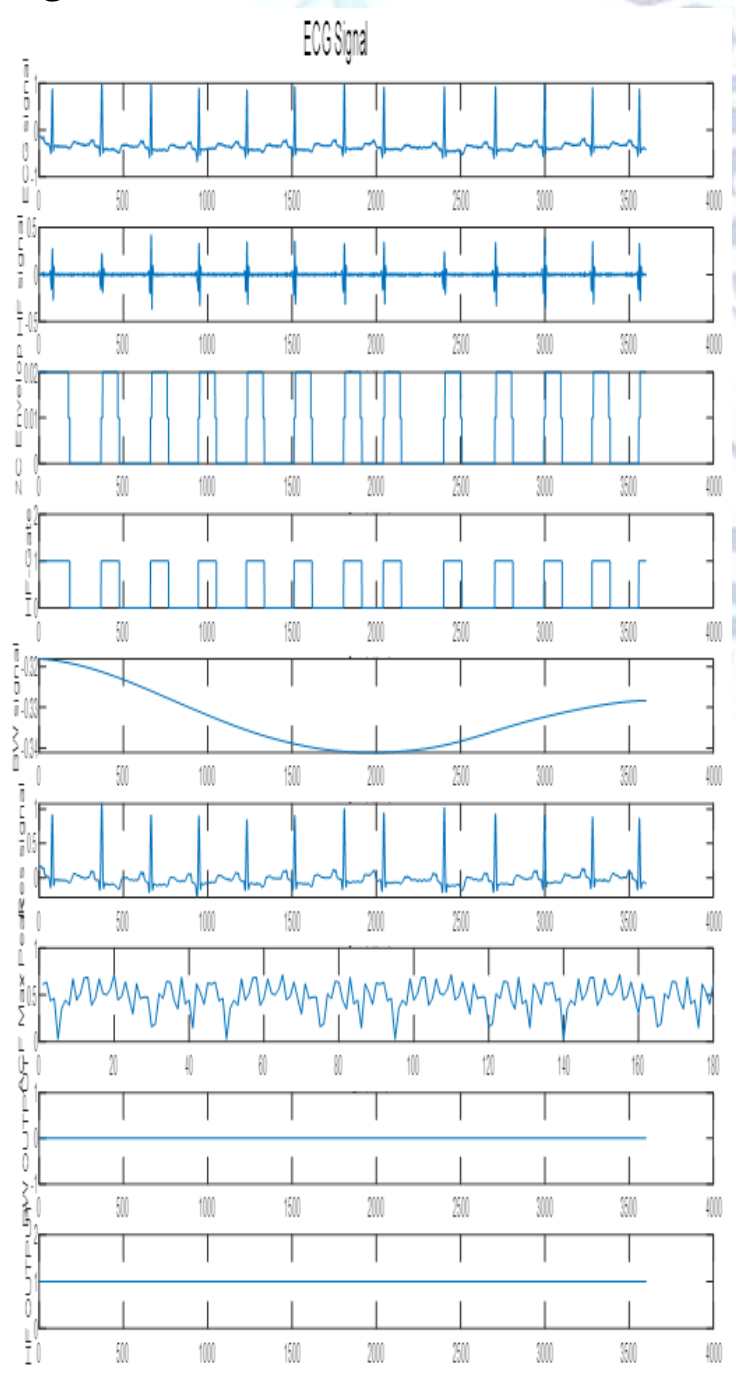

Fig 2. Illustrates the intrinsic mode decomposition functions (IMFs) obtained for the clean and noisy ECG signals. 


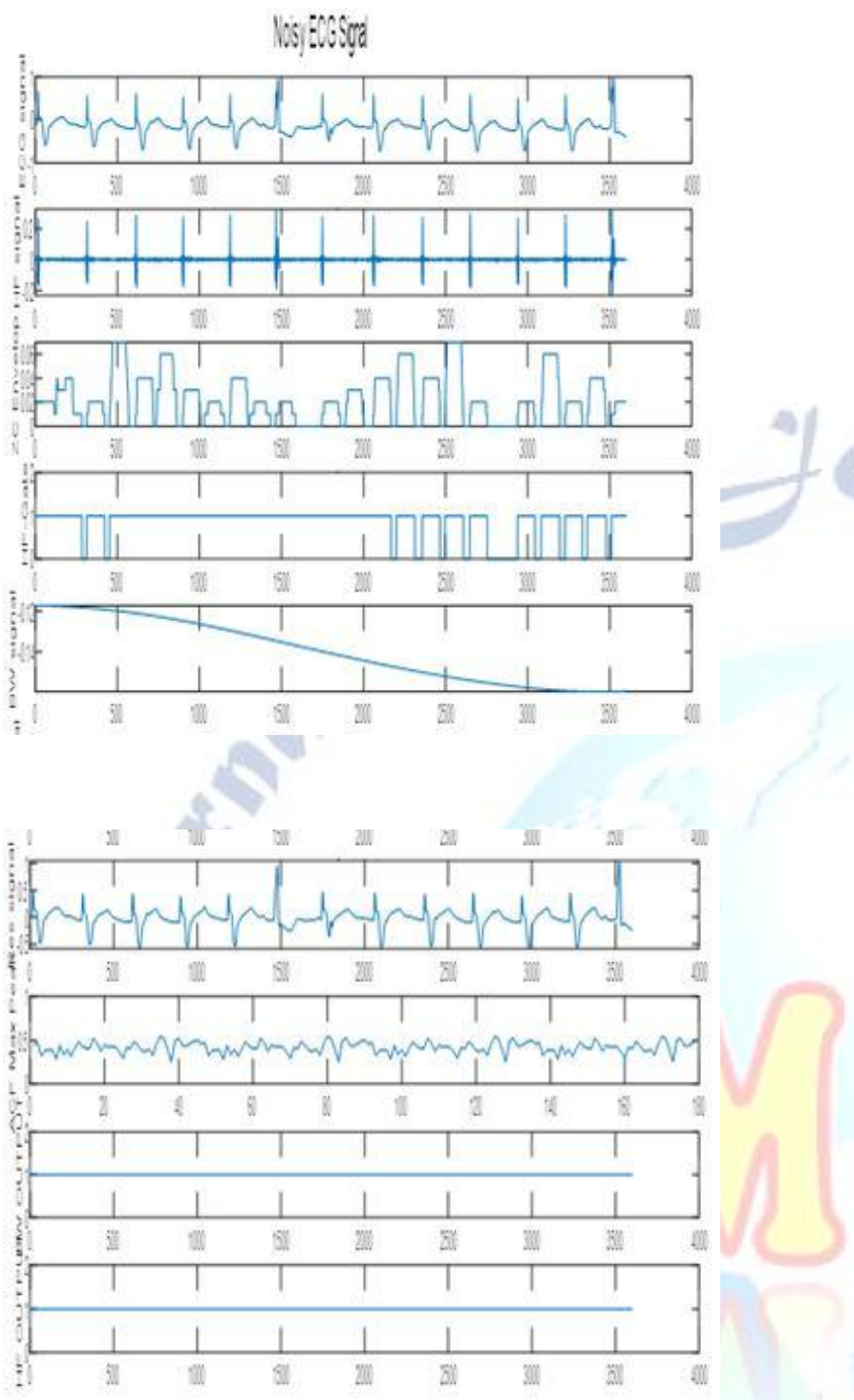

Fig 3. Illustrates the intrinsic mode decomposition functions (IMFs) obtained for the clean and noisy ECG signals

\section{E. Short-term Temporal Feature Extraction}

The ECG noises and artifacts are typically characterized by their peak-to-peak amplitude, frequency content and duration. In this study, we intend to explore the temporal features such as maximum absolute amplitude (MAA), number of zero crossings (NZC) and autocorrelation function $(\mathrm{ACF})$ that are computed from the extracted $\mathrm{HF}$ and LF signals $\mathrm{h}[\mathrm{n}]$ and $\mathrm{b}[\mathrm{n}]$ for detection and classification of different kinds of noises.

\section{F. Noise Detection and Classification Rules}

Based on the aforementioned three temporal features, we build noise detection rules for automatic detection of single and combined noises that are summarized as follows. Baseline Wander Detection Rule: The presence of baseline wander is detected using the temporal features such as MAA and NZC. The BW noise detection rule is defined as

$\mathrm{BW}=\left\{\begin{array}{l}\text { Yes, if }(\max (|\mathrm{b}[\mathrm{n}]|>0.1) \&(\mathrm{NZC}<10) \\ \text { No, otherwise }\end{array}\right.$

For detecting the presence of HF noise, duration of each of the local pulses is compared with the predefined lower and upper duration thresholds. The HF noise detection rule is defined as

HFNoise $=\left\{\begin{array}{l}\text { No; if }(50 \mathrm{~ms}<\text { all widths }<300 \mathrm{~ms} \\ \text { Yes; Otherwise. }\end{array}\right.$

If all the local pulses satisfy the duration thresholds then it is detected as a HF noise-free ECG signal.

Otherwise, the ACF envelope is extracted from the first IMF of the signal. The MA/PLI noise discrimination rule is defined as

HF Noise $=\left\{\begin{array}{l}\text { PLI frame; assign } 1, \text { if } r_{k}>0.7 \\ \text { MA frame; assign } 0, \text { otherwise. }\end{array}\right.$

\section{RESULTS AND DISCUSSION}

In this study, we evaluate performance of the proposed framework using different kinds of noise-free and noisy ECG signals taken from a wide variety of benchmark ECG databases, including the MIT-BIH Arrhythmia (MIT-BIHA) database, the MIT-BIH ST Change (MIT-BIHSTC) database, the PTB Diagnostic ECG (PTBDECG) database, the Fantasia database and the PhysioNet/Computing in Cardiology Challenge 2011 (PCICC2011) database. The MIT-BIHA database contains 48 two-leads ambulatory ECG recordings of half hour each, obtained from 47 subjects. The recordings were digitized at 360 samples per second with 11-bit resolution. The beats, heartbeat type and signal quality were labeled by expert annotators. The MIT-BIHA database contains 15 different types of heartbeats, good quality of ECG signals, signal loss, and different kinds of noises such as baseline wanders and muscle artifacts [34]. The PCICC2011 database contains 2000 twelve-lead ECG recordings (I, II, II, aVR, aVL, aVF, V1, V2, V3, V4, $\mathrm{V} 5$, and V6) with standard diagnostic bandwidth $(0.05-100 \mathrm{~Hz})$. The leads were recorded 
simultaneously for a minimum of 10 seconds and were digitized at 500 samples per second with 16-bit resolution. The ECG signals show different noises and artifacts due to the misplaced electrodes, external interference, poor skin-electrode contact, and artifact resulting from patient motion. The ECG signals were labeled as 'acceptable' or 'unacceptable' by expert annotators. The PTBDECG database contains 549 high-resolution records from 290 subjects. Each record consists of 15-leads, including the 12-leads and the 3 Frank lead ECGs (vx, vy, vz). The signals were digitized at 1000 samples per second with 16 bit resolution. The MIT-BIHSTC database includes 28 two-leads ECG recordings of varying lengths, which were recorded during exercise stress tests. The Fantasia database contains 120 minutes of continuous supine resting ECG signals obtained from 20 young and 20 elderly subjects. Each signal was digitized at $250 \mathrm{~Hz}$ with 12/16-bit resolution. The ECG signals show significant baseline wander and rapid motion artifact noise

\begin{tabular}{|c|c|c|c|c|c|c|c|c|c|c|c|}
\hline Method & $\begin{array}{c}\text { Signal } \\
\text { Group }\end{array}$ & $\begin{array}{c}\text { Total } \\
\text { Segments }\end{array}$ & $\begin{array}{c}\text { Noisy } \\
\text { free } \\
\text { signal }\end{array}$ & $\begin{array}{c}\text { Noisy } \\
\text { Signal }\end{array}$ & TP & TN & FP & FN & Se(\%) & Sp(\%) & OA(\%) \\
\hline \multirow{3}{*}{$\begin{array}{c}\text { Proposed } \\
\text { Method }\end{array}$} & A & 9818 & 1404 & 8414 & 8384 & 1401 & 3 & 30 & 99.6 & 99.7 & 99.6 \\
\cline { 2 - 14 } & B & 800 & 263 & 537 & 528 & 259 & 4 & 9 & 98.3 & 98.4 & 98.3 \\
\cline { 2 - 14 } & C & 450 & 135 & 315 & 313 & 132 & 3 & 2 & 99.3 & 97.7 & 98.8 \\
\cline { 2 - 13 } & D & 3000 & 1500 & 1500 & 1487 & 1473 & 27 & 13 & 99.1 & 98.2 & 98.6 \\
\hline
\end{tabular}

Table 1. Comparison of Noise Detection performance

\section{CONCLUSION}

This paper proposes a novel unified framework for automatic detection, localization and classification of single and combined ECG noises. A modified CEEMD algorithm is presented with new stopping criteria for reducing the computational load. We have explored short-term temporal features, such as maximum absolute amplitude, number of zerocrossings, and local maximum peak of the autocorrelation function for discriminating the BW, MA and PLI noises. Based on the feature thresholding rules, the processed ECG segments are classified into six groups: noise-free ECG, $\mathrm{ECG}+\mathrm{BW}, \mathrm{ECG}+\mathrm{MA}, \mathrm{ECG}+\mathrm{PLI}, \mathrm{ECG}+\mathrm{BW}+\mathrm{PLI}$, and $\mathrm{ECG}+\mathrm{BW}+\mathrm{MA}$.

The proposed method is validated on the large collections of single and multilead ECG signals taken from five standard ECG databases. The proposed method achieves an average Se of
$99.12 \%$, and $\mathrm{Sp}$ of $98.56 \%$, and OA of $98.90 \%$ in detecting the noise-free and noisy ECG signals.

\section{REFERENCES}

[1] Pantelopoulos A, Bourbakis NG. A survey on wearable sensor-based systems for health monitoring and prognosis. IEEE Trans Syst Man Cybern C Appl Rev 2010;40(1):1-12.

[2] Kotas M, Moron T. ECG signals reconstruction in subbands for noise suppression. Biocybern Biomed Eng 2017;37 (December (3)):453-65.

[3] Jekova I, Krasteva V, Christov I, Abdcherli R. Threshold-based system for noise detection in multilead ECG recordings. PhysiolMeas 2012;33(9):1463-77.

[4] Sukor JA. Signal quality measures for pulse oximetry and blood pressure signals acquired in unsupervised home telecare environments, Doc. Dissert.. The Uni. of New South Wales; 2012.

[5] C. Brser et al., "Ambient and unobtrusive cardiorespiratory monitoring techniques," IEEE Reviews Biomed. Eng., vol. 8, pp. 30-43, 2015.

[6] S. Kiranyaz, T. Ince, and M. Gabbouj, "Real-Time patient-specific ECG classification by 1-D convolutional neural networks," IEEE Trans. Biomed. Eng., vol. 63, no. 3, pp. 664-675, March 2016.

[7] U. Satija, B. Ramkumar, and M. S. Manikandan, "Real-Time signal quality-aware ECG telemetry system for IoT-based health care monitoring," IEEE Internet of Things Journal, Feb. 2017.

[8] J. Oster et al., "Semisupervised ECG ventricular beat classification with novelty detection based on switching Kalman filters," IEEE Trans. Biomed. Eng., vol. 62, no. 9, pp. 2125-2134, 2015.

[9] Behar J, Oster J, Li Q, Clifford GD. ECG signal quality during arrhythmia and its application to false alarm reduction. IEEE Trans Biomed Eng 2013;60(6):1660-6.

[10] Orphanidou C, Bonnici T, Charlton P, Clifton D, Vallance D, Tarassenko L. Signal quality indices for the electrocardiogram andphotoplethysmogram: derivation and applications to wireless monitoring. IEEE J Biomed Health Inf 2014;19(3):832-8.

[11] Quesnel PX, Chan ADC, Yang H. Signal quality and false myocardial ischemia alarms in ambulatory electrocardiograms. IEEE Int Symp Med Meas Appl (MeMeA). 2014, June. pp. 1-5.

[12] Hayn D, Jammerbund B, Schreier G. QRS detection based ECG quality assessment. PhysiolMeas 2012;33(9):1449-62.

[13] Johannesen L, Galeotti L. Automatic ECG quality scoring methodology: mimicking human annotators. PhysiolMeas 2012;33(9):1479-90.

[14] Manikandan MS, Ramkumar B. Straightforward and robust QRS detection algorithm for wearable cardiac monitor. Healthcare Technol Lett 2014;1(1):40-4.

[15] Naseri H, Homaeinezhad MR. Electrocardiogram signal quality assessment using an artificially reconstructed target lead. Comput Methods Biomech Biomed Eng 2014;18 (10):1126-41.

[16] Noponen K, Karsikas M, Tiinanen S,Kortelainen J, Huikuri H, Seppnen T. Electrocardiogram quality classification based on robust best subsets linear prediction error. ComputCardiol 2011 Sep;365-8.

[17] Moody BE. Rule-based methods for ECG quality control. ComputCardiol2011;38:361-3. 